\title{
STABLE MANIFOLDS OF HOLOMORPHIC DIFFEOMORPHISMS
}

\author{
MATTIAS JONSSON AND DROR VAROLIN
}

\section{INTRODUCTION}

Let $M$ be a complex manifold and fix once and for all a complete Riemannian metric on $M$ and a holomorphic diffeomorphism, or automorphism, $f \in \operatorname{Diff}_{\mathcal{O}}(M)$. Recall that the stable manifold $W_{p}^{s}$ through a point $p \in M$ with bounded orbit is defined by

$$
W_{p}^{s}:=\left\{x \in M \mid \operatorname{dist}\left(f^{N} x, f^{N} p\right) \leq C \rho^{N} \text { for } N \geq 0\right\},
$$

where $\rho=\rho_{p}<1$ and $C=C_{p}>0$. It turns out that often $W_{p}^{s}$ is an immersed complex manifold. Assuming this to be the case, the following problem was posed by E. Bedford [B].

Problem: Determine the complex structure of the stable manifolds of $f$.

In many cases it can be shown that $W_{p}^{s}$ is a monotone union of balls, and this in turn implies Br] that it is diffeomorphic to real Euclidean space. Moreover, by the contracting nature of the dynamics, one sees that the Kobayashi pseudometric of $W_{p}^{s}$ vanishes identically. However, when $\operatorname{dim}\left(W_{p}^{s}\right) \geq 3$, it is not possible to deduce only from these properties that $W_{p}^{s}$ is biholomorphic to Euclidean space. For example, there exist monotone unions of balls which are not Stein [F]. (The question of Steinness of monotone unions of balls in complex dimension 2 is open.) When $\operatorname{dim}\left(W_{p}^{s}\right)=1$, the Uniformization Theorem implies that $W_{p}^{s}$ is biholomorphic to $\mathbf{C}$ [BLS, W].

The main results of this paper are proved in the non-uniform setting, i.e. with respect to compactly supported invariant measures. More precisely, we say that a subset $A \subset M$ is invariant if $f A=A$, and that it has total measure if $\mu(A)=1$ for every compactly supported invariant probability measure $\mu$.

Our main objective in this paper is to prove the following theorem.

Theorem 1. There exists an invariant Borel set $\mathcal{K}(f) \subset M$ of total measure such that for every $p \in \mathcal{K}(f), W_{p}^{s}$ is a complex manifold biholomorphic to complex Euclidean space.

Let us clarify things a little further. The set $\mathcal{K}(f)$ is the set of so-called Oseledec points or regular points. Its existence is a part of the well known and fundamental theorem of V. Oseledec [O]. The equally fundamental work of Pesin $[\mathrm{P}]$ states in part that the stable manifold passing through each Oseledec point $p \in \mathcal{K}(f)$ is an immersed (complex) manifold. What we show is that for each $p \in \mathcal{K}(f), W_{p}^{s}$ is biholomorphic to $\mathbf{C}^{k}$, where $k=\operatorname{dim}_{\mathbf{C}} W_{p}^{s}$.

We postpone to Section 3 a more detailed discussion of the results of Oseledec and Pesin which we will use in this paper. For now, however, we content ourselves with saying that every point in $\mathcal{K}(f)$ has a bounded orbit, although $\mathcal{K}(f)$ itself need not be bounded. Moreover, we emphasize that, to have a non-trivial result, it is necessary to have at least one invariant measure with compact support, but this is guaranteed to happen once $f$ leaves invariant a bounded subset of $M$.

Our approach to proving Theorem 1 is to associate to the dynamical system $f$ a certain "unraveled" dynamical system, and then conjugate the latter to a much simpler (polynomial) dynamical system on the so-called stable distribution. To state the result more precisely we need to develop some notation and concepts, which we now proceed to do.

Date: October 27, 2018. 
Recall that the stable distribution $E^{s}$ is a family of vector subspaces $E_{p}^{s}$ of $T M_{p}$ on which $d f$ is asymptotically contracting: for $p \in M$ with bounded orbit, $E_{p}^{s}$ is given by

$$
E_{p}^{s}:=\left\{v \in T M_{p}|| d f^{N} v \mid \leq C \rho^{N} \text { for } N \geq 0\right\},
$$

where $\rho=\rho_{p}<1$ and $C=C_{p}>0$. Notice that this exponential decay is not uniform, i.e. $p \mapsto \rho_{p}$, $p \mapsto C_{p}$ and even $p \mapsto \operatorname{dim} E_{p}^{s}$ could be discontinuous.

From here on, let $\mathcal{K}(f)$ denote the set of Oseledec points (whose well known definition is recalled in Section (3). If the stable manifold $W_{p}^{s}$ defined above exists as an immersed submanifold of $M$, then $\left(T W_{p}^{s}\right)_{p}=E_{p}^{s}$. In view of Pesin's work mentioned above, this is the case whenever $p \in \mathcal{K}(f)$.

Next we discuss what was meant by "unraveled" above. To this end, even though it might happen that for $q \neq p$ the two stable manifolds $W_{p}^{s}$ and $W_{q}^{s}$ intersect and thus agree, we treat $W_{p}^{s}$ and $W_{q}^{s}$ as distinct stable manifolds. More precisely, we define the set

$$
\mathcal{W}^{s}:=\bigsqcup_{p \in \mathcal{K}(f)} W_{p}^{s} .
$$

Since $W_{f p}^{s}=f W_{p}^{s}, f$ induces a bijection on $\mathcal{W}^{s}$, still denoted $f$, which is holomorphic on the fibers. (We will topologize $\mathcal{W}^{s}$ and $E^{s}$ shortly.) We note again that the bundle $\mathcal{W}^{s}$ is defined only over the topological space $\mathcal{K}(f)$ of Oseledec points. The following is our main result.

Theorem 2. There exists a measurable isomorphism $\Psi: \mathcal{W}^{s} \rightarrow E^{s} \mid \mathcal{K}(f)$ and a bundle automorphism $P: E^{s}\left|\mathcal{K}(f) \rightarrow E^{s}\right| \mathcal{K}(f)$ such that, for every $p \in \mathcal{K}(f)$,

1. $P_{p}: E_{p}^{s} \rightarrow E_{f p}^{s}$ is a polynomial automorphism;

2. $P_{p}^{N} \rightarrow 0$ locally uniformly on $E_{p}^{s}$;

3. $\Psi_{p}:=\Psi \mid W_{p}^{s}$ is a biholomorphism of $W_{p}^{s}$ onto $E_{p}^{s}$ and $\left(d \Psi_{p}\right)_{p}=\mathrm{id}$;

4. $\Psi \circ f \circ \Psi^{-1}=P$ on $\mathcal{K}(f)$.

Remark. Note first that (3) implies Theorem 1. Secondly, we do not claim that $p \mapsto \operatorname{deg}\left(P_{p}\right)$ is constant or even bounded. However, it is constant along orbits. Finally, the map $\Psi$ turns out to be slightly better than measurable. It is slowly varying; a concept we shall discuss more thoroughly in Section 1 .

We now return to the question of topologizing $\mathcal{W}^{s}$ and $E^{s}$. Let

$$
\begin{gathered}
\operatorname{dist}(x, y)=\left\{\begin{array}{r}
x, y \in W_{p}^{s} \\
\operatorname{dist}_{p}(x, y) \\
\operatorname{dist}_{p}(x, p)+\mathrm{d}(p, q)+\operatorname{dist}_{q}(q, y) \quad x \in W_{p}^{s}, y \in W_{q}^{s}
\end{array}\right. \\
\operatorname{dist}(v, w)= \begin{cases}|v-w| & v, w \in E_{p}^{s} \\
|v|+\mathrm{d}(p, q)+|w| & v \in E_{p}^{s}, w \in E_{q}^{s}\end{cases}
\end{gathered}
$$

The function $\operatorname{dist}_{p}$ is a distance on $W_{p}^{s}$ associated to the complete Riemann metric on $M$ (so that dist $_{p}$ recovers the intrinsic topology of $W_{p}^{s}$ ). The function d appearing on the right hand side of these definitions is the same for both functions. In the case of Theorem 2 we shall take $\mathrm{d}(p, q)=\delta_{p, q}$, i.e. $\mathcal{W}^{s}$ is the disjoint union of all the stable manifolds. We refer to this as the discrete case. Later on, it is also useful to take $\mathrm{d}$ to be the Riemannian distance on $M$. We shall refer to this as the bouquet case. To these topologies we associate the Borel sets, and it is with respect to this $\sigma$-algebra that $\Psi$ is measurable.

We turn now to the hyperbolic picture. Recall that $f \in \operatorname{Diff}_{\mathcal{O}}(M)$ is hyperbolic on a compact set $K$ if $K$ is invariant and there exists a continuous splitting

$$
\left.T M\right|_{K}=E^{s} \oplus E^{u}
$$

with the following properties.

(a) $E_{p}^{s}$ and $E_{p}^{u}$ have constant rank for all $p \in K$, say $k$ and $n-k(n=\operatorname{dim} M)$;

(b) $d f\left(E_{p}^{s}\right)=E_{f p}^{s}$ and $d f\left(E_{p}^{u}\right)=E_{f p}^{u}$ for all $p \in K$; 
(c) there exist positive constants $C$ and $\rho$, with $\rho<1$, so that, for all $p \in K$ and all $N \geq 0$

$$
\left\|d f^{N} \mid E_{p}^{s}\right\| \leq C \rho^{N} \quad \text { and } \quad\left\|d f^{N} \mid E_{p}^{u}\right\| \geq C^{-1} \rho^{-N} .
$$

In this case we write

$$
\mathcal{W}^{s}=\bigsqcup_{p \in K} W_{p}^{s} \quad \text { and } \quad E^{s}=\bigcup_{p \in K} E_{p}^{s}
$$

Then $E^{s}$ is a continuous vector bundle and the set of (local) stable manifolds form a lamination near $K$ (see, e.g. [S]). (We are abusing language slightly here: we are interested in the restriction of $E^{s}$ to the set $K$ of hyperbolicity, but $E^{s}$ is being written instead of $E^{s} \mid K$.)

Theorem 11 implies that $W_{p}^{s}$ is biholomorphic to $\mathbf{C}^{k}$ for every $p \in \mathcal{K}(f) \cap K$. Moreover there are always invariant measures on an invariant compact set, so the set $\mathcal{K}(f) \cap K$ is nonempty: it contains every periodic point in $K$ and many more points (unless $K$ is a finite set). Nevertheless, one would like to prove that every stable manifold through $K$ is biholomorphic to $\mathbf{C}^{k}$. We conjecture that this is indeed the case. The main problem in proving this is that even though $d f^{N}$ uniformly contracts vectors in $E_{p}^{s}, p \in K$, the exact rate of contraction can be highly nonconstant. In fact, controlling the (asymptotic) rates of contraction within $E^{s}$ is central to our approach.

On the other hand, the conjecture above is easy to prove if an (unfortunately quite strong) hypothesis is placed on the map $f$. If $A$ is the restriction to $E^{s}$ of $d f$, let

$$
L_{+}:=\limsup _{N \rightarrow \infty} \sup _{p \in K} N^{-1} \log \left\|A_{p}^{N}\right\| \quad \text { and } \quad L_{-}:=\liminf _{N \rightarrow \infty} \inf _{p \in K}-N^{-1} \log \left\|A_{p}^{-N}\right\| .
$$

Note that $L_{-} \leq L_{+}<0$. We say that $f$ is equi-contracting if $2 L_{+}<L_{-}$.

Note, in particular, that if $f$ has one dimensional stable manifolds, then $f$ is automatically equicontracting.

Theorem 3. If $f$ is hyperbolic and equi-contracting on a compact set $K$, then there exists a homeomorphism $\Psi: \mathcal{W}^{s} \rightarrow E^{s}$ over $K$ such that

1. for every $p \in K, \Psi_{p}:=\Psi \mid W_{p}^{s}$ is a biholomorphism of $W_{p}^{s}$ onto $E_{p}^{s}$ and $\left(d \Psi_{p}\right)_{p}=\mathrm{id}$;

2. $\Psi \circ f \circ \Psi^{-1}=d f \mid E^{s}$.

In Theorem 3 we use the bouquet topology on $\mathcal{W}^{s}$ and $E^{s}$. Note that the equi-contracting hypothesis implies that $f$ can be brought to a linear form as opposed to the more general polynomial form given by Theorem 2 .

Regarding history, while Oseledec/Pesin theory has been used in complex dynamics before (see e.g. [BD], [BLS]), to our knowledge this is the first application to the study of the complex structure of stable manifolds in higher dimensions. When $f$ has a fixed point, the fact that $f$ is conjugate to a normal form is due to S. Sternberg [St], and (independently, though much later) to Rosay and Rudin $[\mathrm{RR}$ in the holomorphic case with essentially the same proof. (We note that, even though Sternberg's theorem is stated for an attracting fixed point, say $p$, one can reduce to this case by restricting $f$ to its stable manifold $W_{p}^{s}$, since the latter is invariant in the fixed point case.) The condition for linearization was known to C. Siegel [Si]. In the general, non-stationary case, very little seems to have been done. The main work we know of is due to M. Guysinsky and A. Katok [G, GK]. However, they place rather strong hypotheses on the spectrum of the of $d f$ which, while sufficient (and perhaps more so necessary) for their applications, would be much too strong for the problem we are interested in here.

Roughly speaking, our approach combines the ideas from the proof of Sternberg's Theorem with techniques from Oseledec-Pesin Theory. The proof of Sternberg's theorem, as in St or [RR], uses linear algebra to split the stable space $E_{p}^{s}$ into invariant subspaces where $d f$ has an essentially fixed rate of contraction (given by the eigenvalues of $d f \mid E_{p}^{s}$ ). In the setting of Theorem 2 we use Oseledec-Pesin theory in order to control the rate of contraction of $d f$. 
The organization of the paper is as follows. In Section 1 we define a continuous family of uniformly sized charts for the stable manifolds, and in Section 2 we prove Theorem 3. In Section 3 we state the results we use from the Oseledec/Pesin theory and in Section 1 we set up the slowly varying formalism, working out some useful lemmas and propositions. In Section 5 we prove the existence of $\Psi$ locally. This section is the main step in the proof of Theorem 2, the latter being completed in Section 6 .

Acknowledgments We thank John-Erik Fornæss and Ralf Spatzier for their interest in this project and for interesting discussions, and more particularly we thank Ralf for also directing us to many useful references on related results in real dynamics. We are also grateful to Charles Favre, Nessim Sibony and the referee for many helpful comments and suggestions.

\section{Holomorphic exponential maps I. Hyperbolic CASE}

In this section, we construct a continuous (in $p$ ) family of biholomorphic maps $\chi_{p}$ from a neighborhood of $0_{p}$ in $E_{p}^{s}$ into $W_{p}^{s}$. To this end, let $f \in \operatorname{Diff}_{\mathcal{O}}(M)$ be hyperbolic on a compact set $K \subset \subset M$. For $\epsilon>0$, set

$$
E_{p}^{s}(\epsilon):=\left\{v \in E_{p}^{s}|| v \mid<\epsilon\right\} \quad \text { and } \quad E^{s}(\epsilon):=\bigcup_{p \in K} E_{p}^{s}(\epsilon),
$$

the latter equipped with the bouquet topology discussed in the Introduction.

Proposition 1.1. There exists $\epsilon>0$ and a continuous mapping $\chi: E^{s}(\epsilon) \rightarrow \mathcal{W}^{s}$ which maps each $E_{p}^{s}(\epsilon)$ biholomorphically into $W_{p}^{s}$, maps the zero vector $0_{p} \in E_{p}^{s}$ to $p \in W_{p}^{s}$, and satisfies $d\left(\chi \mid E_{p}^{s}\right)_{0_{p}}=\operatorname{id}_{E_{p}^{s}}$.

Remark. Originally, we had a rather complicated and not even completely general proof of this proposition. We thank C. Favre for showing us a much simpler and complete proof, which we now present.

Proof of Proposition 1.1. As mentioned before, it is shown in [S] that $\mathcal{W}^{s}$ gives a lamination near $K$. Cover $K$ by a finite number of balls $B_{i}, 1 \leq i \leq l$. Let

$$
\xi_{i}: E^{s}\left(\epsilon_{i}\right) \mid B_{i} \cap K \rightarrow \mathcal{W}^{s}, \quad 1 \leq i \leq l
$$

be local parameterizations of $\mathcal{W}^{s}$ near $K \cap B_{i}$ such that, for all $p \in B_{i} \cap K, \xi_{i}\left(0_{p}\right)=p$ and $d \xi_{i}\left(0_{p}\right)=i d$. Such parameterizations exist if the balls $B_{i}$ are taken small enough. Write $\epsilon:=\min \left\{\epsilon_{i} ; 1 \leq i \leq l\right\}$. Let $\mathcal{W}_{i}^{s}(\epsilon)=\xi_{i}\left(E^{s}(\epsilon) \mid B_{i} \cap K\right)$ be the image of $E^{s}(\epsilon) \mid B_{i} \cap K$ under $\xi_{i}$, and set $\mathcal{W}^{s}(\epsilon)=\cup_{i} \mathcal{W}_{i}^{s}(\epsilon)$. Finally, let $\left\{\varphi_{i} ; 1 \leq i \leq l\right\}$ be a partition of unity subordinate to the covering $\left\{B_{i} ; 1 \leq i \leq l\right\}$ of $K$. Then the map $\chi: E^{s}(\epsilon) \rightarrow \mathcal{W}^{s}(\epsilon)$ whose inverse is given by the formula

$$
\chi^{-1}(x)=\sum_{1 \leq i \leq l} \varphi_{i}(p) \xi_{i}^{-1}(x), \quad x \in W_{p}^{s},
$$

has the desired properties.

\section{Proof of Theorem 3}

We first sketch the basic idea of the proof. Let

$$
A:=d f \mid E^{s}
$$

denote the restriction to $E^{s}$ of $d f$ and, with $\chi$ as in Proposition 1.1, set

$$
F:=\chi^{-1} \circ f \circ \chi \text { and } \mathcal{W}^{s}(\epsilon):=\chi\left(E^{s}(\epsilon)\right) \text {. }
$$

Here and below, in order to avoid referring to the specific coordinates $v$ chosen on $E^{s}$, we use the notation $O(m)$ in place of the more common $O\left(|v|^{m}\right)$. 
We want to show that the map $A^{-N} \chi^{-1} f^{N}$ converges, locally uniformly on $\mathcal{W}^{s}$ as $N \rightarrow \infty$, to a biholomorphic map. (Note that, because of the use of $\chi^{-1}$, the former map is only defined on some compact subset of $\mathcal{W}^{s}$.) Since $A^{-1} F-$ id $=O(2)$, we have, on a given compact subset of $\mathcal{W}^{s}$ and for $N$ sufficiently large, that

$$
\begin{aligned}
A^{-(N+1)} \chi^{-1} f^{N+1}-A^{-N} \chi^{-1} f^{N} & =A^{-N}\left(A^{-1} F-\mathrm{id}\right) \chi^{-1} f^{N} \\
& \sim e^{\left(-L_{-}+2 L_{+}\right) N},
\end{aligned}
$$

where the last estimate is uniform on compact sets. By the equi-contracting hypothesis, this implies locally uniform convergence. Injectivity and surjectivity of the limit map are then easily established. The details are as follows.

Given $\delta>0$ with $L_{+}+\delta<0$ there exists $N_{0} \in \mathbf{N}$ and $\epsilon>0$ such that

$$
\left|F^{N_{0}} v\right| \leq e^{\left(L_{+}+\delta\right) N_{0}}|v| \text { whenever } v \in E^{s}(\epsilon) .
$$

This follows from the definition of $L_{+}$and the fact that $\left(d F_{p}\right)_{p}=A_{p}$. Now set

$$
C:=\sup \left\{\left|F^{j} v\right| /|v| ; 0 \leq j<N_{0}, v \in E^{s}(\epsilon)\right\} .
$$

For $N \geq 0$, write $N=k N_{0}+j$ with $0 \leq j<N_{0}$. Then

$$
\left|F^{N} v\right|=\left|F^{j}\left(F^{k N_{0}} v\right)\right| \leq C\left|F^{k N_{0}} v\right| \leq C e^{\left(L_{+}+\delta\right) k N_{0}}|v|,
$$

and so there exists $N_{1}=N_{1}(\delta)$ such that for all $N \geq N_{1}$,

$$
\left|F^{N} v\right| \leq e^{\left(L_{+}+2 \delta\right) N}, \quad v \in E^{s}(\epsilon)
$$

Now consider a compact $J \subset \subset \mathcal{W}^{s}$. By the contracting nature of $f$ there exists $n \geq 0$ such that $f^{n}(J) \subset \mathcal{W}^{s}(\epsilon)$. Since $\chi^{-1}$ is continuous on $\mathcal{W}^{s}(\epsilon)$, the above estimate implies that there exists $N_{2}=N_{2}(J, \delta) \geq N_{1}+n$ such that for all $N \geq N_{2}$,

$$
\left|\chi^{-1} f^{N} z\right| \leq e^{\left(L_{+}+3 \delta\right) N}, \quad z \in J
$$

Since $\left(d F_{p}\right)_{p}=A_{p}$ there exists $C>0$ such that for all $v \in E^{s}(\epsilon)$,

$$
\left|v-A^{-1} F v\right| \leq C|v|^{2} \text {. }
$$

Using the definition of $L_{-}$and increasing $N_{2}$ if necessary, one then obtains, for all $z \in J$ and $N \geq N_{2}$, the estimate

$$
\begin{aligned}
\left|A^{-N} \chi^{-1} f^{N} z-A^{-(N+1)} \chi^{-1} f^{N+1} z\right| & \leq\left\|A^{-N}\right\| \cdot\left|w_{N}-A^{-1} F w_{N}\right|^{2} \\
& \leq e^{-\left(L_{-}-\delta\right) N} C\left|w_{N}\right|^{2} \\
& \leq C e^{\left(2 L_{+}-L_{-}+5 \delta\right) N},
\end{aligned}
$$

where $w_{N}=\chi^{-1} f^{N} z$. Since $2 L_{+}<L_{-}$by the equi-contracting hypothesis, it follows that

$$
\Psi:=\lim _{N \rightarrow \infty} A^{-N} \chi^{-1} f^{N}
$$

exists locally uniformly in the bouquet topology. Thus $\Psi$ is continuous and clearly satisfies $d \Psi=\mathrm{id}$ as well as the functional equation

$$
\Psi \circ f \circ \Psi^{-1}=A .
$$

We claim that $\Psi$ is in fact a homeomorphism. Clearly $\Psi\left(W_{p}^{s}\right) \subset E_{p}^{s}$ for all $p \in K$. For fixed $p, \Psi \mid W_{p}^{s}$, being a uniform limit of automorphisms, is holomorphic and injective. Thus $\Psi$ is itself injective. This implies that $\Psi\left(\mathcal{W}^{s}\right)$ contains a neighborhood $\mathcal{N}$ of the zero section of $E^{s}$. We now use the contracting property of $A$ to show surjectivity of $\Psi$. Consider any $v \in E_{p}^{s}$ and pick $N$ large enough so that $A^{N} v \subset \mathcal{N}$, i.e. there exists $y \in W_{f^{N} p}^{s}$ with $\Psi(y)=A^{N} v$. Let $x:=f^{-N} y$. Then

$$
v=A^{-N} \Psi(y)=\Psi\left(f^{-N} y\right)=\Psi(x) .
$$


Thus $\Psi$ is a homeomorphism, which completes the proof.

\section{LYAPUNOV DATA AND STABLE MANIFOLDS}

In this section, we give an overview, containing no proofs, of various results in smooth ergodic theory. There are several references which the reader can consult for details. We have taken most of our statements from [PS], but a more detailed proof of some of the theorems can be found in [M].

First, to an automorphism $f$ one can associate its Lyapunov data: these are vector spaces $E_{p}^{\lambda} \subset$ $T M_{p}$, called the Lyapunov spaces of $f$, defined by

$$
E_{p}^{\lambda}:=\left\{v \in T M_{p}\left|\lim _{N \rightarrow \pm \infty} N^{-1} \log \right| d f^{N} v \mid=\lambda\right\} .
$$

The numbers $\lambda=\lambda(p)$ such that $E_{p}^{\lambda} \neq\{0\}$ are called the Lyapunov exponents.

In general, of course, there are only a finite number of Lyapunov exponents at a given point $p$. A point $p$ with bounded orbit such that

$$
T M_{p}=\bigoplus_{\lambda \in \mathbf{R}} E_{p}^{\lambda}
$$

is called an Oseledec point (or regular point) of $f$. The splitting (3.1) is called the Lyapunov splitting. We denote the set of Oseledec points by $\mathcal{K}(f)$.

Before stating the basic result on Oseledec points, we need the following definitions.

Definition 3.1. Let $X \subset M$ be an $f$-invariant Borel set.

1. A Borel function $R: X \rightarrow(0, \infty)$ is called $\epsilon$-slowly varying if $e^{-\epsilon} \leq R(f p) / R(p) \leq e^{\epsilon}$ for every $p \in X$.

2. A collection of Borel functions $\left\{R_{\epsilon}: X \rightarrow(0, \infty) ; \epsilon>0\right\}$ is called a slow variation if $R_{\epsilon}$ is $\epsilon$-slowly varying for every $\epsilon>0$.

3. A function $h: X \rightarrow(0, \infty)$ is called slowly varying if there exists a slow variation $R_{\epsilon}$ such that either $h \leq R_{\epsilon}$ or $h \geq 1 / R_{\epsilon}$ for all $\epsilon>0$.

Remark. In what follows, we shall have to control either the growth or shrinking of certain functions along orbits of $f$. To distinguish these two situations, we establish the following convention: in the former case, the functions shall have ranges of the form $(a, \infty)$ with $a \geq 1$, and in the latter, ranges of the form $(0, b)$ with $b<\infty$.

Theorem $3.2([\mathrm{G}])$. The set $\mathcal{K}(f)$ is an invariant Borel set of total measure. Moreover, there is a slow variation $\left\{R_{\epsilon}: \mathcal{K}(f) \rightarrow(1, \infty)\right\}$ such that for all $p \in \mathcal{K}(f)$ and all $\epsilon>0$,

a)

$$
R_{\epsilon}(p)^{-1} e^{-\epsilon N} \leq \frac{\left|d\left(f^{N}\right)_{p} v\right|}{e^{\lambda N}|v|} \leq R_{\epsilon}(p) e^{\epsilon N} \quad \text { whenever } \quad v \in E_{p}^{\lambda}
$$

b)

$$
\measuredangle\left(E_{p}^{\lambda}, E_{p}^{\lambda^{\prime}}\right) \geq \frac{1}{R_{\epsilon}(p)} \text { whenever } \lambda^{\prime} \neq \lambda .
$$

At every Oseledec point, one has the following decomposition.

$$
T M_{p}=E_{p}^{s} \oplus E_{p}^{0} \oplus E_{p}^{u},
$$

where

$$
E_{p}^{s}=\bigoplus_{\lambda<0} E_{p}^{\lambda} \quad \text { and } \quad E_{p}^{u}=\bigoplus_{\lambda>0} E_{p}^{\lambda}
$$


Given such a $p$ we define the stable manifold at $p$ by

$$
W_{p}^{s}:=\left\{\begin{array}{l|l|l}
x \in M & \limsup _{N \rightarrow \infty} \frac{1}{N} \log \operatorname{dist}\left(f^{N} x, f^{N} p\right)<0
\end{array} .\right.
$$

The Pesin stable manifold theorem can thus be stated as follows.

Theorem $3.3([\mathrm{P}])$. For every $p \in \mathcal{K}(f), W_{p}^{s}$ is an immersed (complex) submanifold of $M$.

In fact, Pesin's result also tells us how the stable manifolds $W_{p}^{s}$ depend on the base point $p$. In particular we have a non-uniform version of the exponential map in Proposition 1.1 .

Theorem $3.4([\mathrm{P}])$. The stable lamination $\mathcal{W}^{s}$ defined by $(0.1)$ is a slowly varying lamination on $\mathcal{K}(f)$ in the following sense: let $M \hookrightarrow \mathbf{R}^{n}$ be an isometric immersion into Euclidean space. Then there is a slowly varying function $r: \mathcal{K}(f) \rightarrow(0,1)$ with the property that if $D_{p}$ is the ball of radius $r(p)$ and center $0_{p}$ in $E_{p}^{s}$, then the orthogonal projection $\Pi: W_{p}^{s} \rightarrow E_{p}^{s}$ is invertible on the branch of $\Pi^{-1}\left(D_{p}\right)$ containing $p$. Moreover, there exists a map $\chi: E^{s}(r) \rightarrow \mathcal{W}^{s}$ which maps $E_{p}^{s}(r(p))$ biholomorphically into $W_{p}^{s}$, maps $0_{p}$ to $p$, and satisfies $d \chi_{0_{p}}=\operatorname{id}_{E_{p}^{s}}$. Moreover, $p \mapsto\left\|d \chi_{p} \mid E^{s}(r(p))\right\|$ is a slowly varying function on $\mathcal{K}(f)$.

Remark. This lamination aspect of Pesin's theorem is rarely stated, but it is easily seen to be true if one follows the proof, say in [PS], based on the graph transform.

The set $\mathcal{K}(f)$ can be further decomposed into invariant subsets as follows. For $l \in \mathbf{N}_{+}, \underline{\lambda}=$ $\left(\lambda_{1}, \ldots, \lambda_{l}\right)$ with $\lambda_{l}<\cdots<\lambda_{1}<0$ and $\underline{m}=\left(m_{1}, \ldots, m_{l}\right)$, let

$$
\mathcal{K}(\underline{\lambda}, \underline{m}):=\left\{p \in \mathcal{K}(f) \mid E_{p}^{s}=E_{p}^{\lambda_{1}} \oplus \cdots \oplus E_{p}^{\lambda_{l}} \text { and } \operatorname{dim}\left(E_{p}^{\lambda_{j}}\right)=m_{j} 1 \leq j \leq l .\right\} .
$$

Then

$$
\mathcal{K}(f)=\bigcup_{\underline{\lambda}, \underline{m}} \mathcal{K}(\underline{\lambda}, \underline{m}),
$$

and each $\mathcal{K}(\underline{\lambda}, \underline{m})$ is a Borel set which is invariant for $f$. These subset of "constant stable Lyapunov data" will be crucial to our further analysis.

\section{Slowly VARYing BUNDLES AND MAPS}

In this section we establish definitions and basic results about slowly varying objects. The slowly varying notion of regularity is the strongest form of regularity that can be expected to hold in the non-uniform picture. Roughly speaking, slowly varying objects can be treated as constants, as long as we are interested in exponential estimates.

Measurable bundles and maps. Recall that the relative $k$-Grassmannian of $T M$ is a bundle $\mathcal{G}_{k}(T M) \rightarrow M$ whose fiber over $p \in M$ is the set of $k$ dimensional complex subspaces of $T M_{p}$. A measurable complex vector bundle over a Borel subset $X \subset M$ is then a measurable section $E$ of the Grassmann bundle $\mathcal{G}_{k}(T M)$ over $X$. A measurable subbundle $E^{\prime}$ of a measurable complex vector bundle $E$ is a measurable complex vector bundle such that $E_{p}^{\prime}$ is a subspace of $E_{p}$ for each $p \in X$. We implicitly assume that the base $X$ is invariant for $f$, and endow all such vector bundles with the metric inherited from $T M$. The total spaces are given the discrete topology discussed in the introduction.

As a matter of notation, given a function $g: X \rightarrow(0, \infty)$, let

$$
E(g):=\bigcup_{p \in X} E_{p}(g(p))
$$

where $E_{p}(r)=\left\{v \in E_{p}|| v \mid<r\right\}$. Notice that this is a neighborhood of the zero section $O_{E}$ of $E$ and we call such a neighborhood a tube. Later on we will work with slowly varying tubes, i.e. tubes defined by slowly varying functions $g$. 
Let $E \rightarrow X$ and $E^{\prime} \rightarrow X$ be two measurable vector bundles. A (measurable) bundle map $\Phi: E \rightarrow E^{\prime}$ fibered over $\phi: X \rightarrow X$ is then a map of the total spaces, defined in some tube $E(g)$, such that $\phi$ is measurable (with respect to the Borel $\sigma$-algebra inhereted from $M$ ) and $\Phi\left(E_{p}\right) \subset E_{\phi(p)}^{\prime}$ for $p \in X$. We shall require that $\Phi$ map the zero section of $E$ to the zero section of $E^{\prime}$, and that the map $\Phi_{p}:=\Phi \mid E_{p}^{\prime}$ be holomorphic for each $p \in X$. This situation is sometimes denoted by the shorthand $\Phi / \phi$. In our setting, it will always be the case that $\phi=f$ or $\phi=$ id.

A bundle map $\Phi / \phi$ is said to be homogeneous of degree $m$ if for every $c \in \mathbf{C}$ one has $\Phi(c \cdot v)=$ $c^{m} \cdot \Phi(v)$. We use the notation

$$
\left\|\Phi_{p}\right\|:=\max _{v \in E_{p}} \frac{\left|\Phi_{p}(v)\right|}{|v|^{m}}
$$

and the notation $\|\Phi\|: p \mapsto\left\|\Phi_{p}\right\|$.

More generally, $\Phi$ is said to be polynomial of degree $m$ if there exist homogeneous maps $\Phi_{j}$ of degree $j, 1 \leq j \leq m$ such that $\Phi=\sum_{j=1}^{m} \Phi_{j}$. By our requirement that the fiber maps be holomorphic, every bundle map $\Phi / \phi$ has a homogeneous expansion

$$
\Phi=\sum_{m=1}^{\infty} \Phi_{m}
$$

Using the notation in (4.1) we have $B(p):=\sup _{m}\left\|\Phi_{m, p}\right\|^{1 / m}<\infty$ for every $p \in X$. In general one can say very little about the dependence of $B(p)$ on $p$, but the maps we will work with have more regularity: we say that $\Phi$ is slowly varying if $B$ is slowly varying in the sense of Definition 3.1.

It trivially follows that if $\Phi$ is slowly varying, then so are all of its homogeneous parts $\Phi_{m}$. Also, sums, compositions and inverses of slowly varying maps are easily seen to be slowly varying.

Tubes associated to slowly varying maps. Consider a slowly varying selfmap $T$ /id of a bundle $E \rightarrow X$ such that $d T \mid O_{E}=$ id. This has the homogeneous expansion

$$
T=\mathrm{id}+\sum_{j=2}^{\infty} T_{j}
$$

Then $\left\|T_{j}\right\| \leq B^{j}$ for some slowly varying function $B: X \rightarrow(2, \infty)$ and $T$ is defined on the slowly varying tube $E(1 / B)$.

In the next two propositions, which will be crucial in the final step of the proof of Theorem 2, we will show that the range, as well as the domain of injectivity of $T$ contain slowly varying tubes.

Proposition 4.1. Let $T / \mathrm{id}$ be as above. Then there exists a slowly varying function $g: X \rightarrow(0,1)$ such that

$$
T(E(1 / B))) \supset E(g) .
$$

Proof. For each $v \in E(1 / B)$, one has

$$
|T v| \geq|v|-\sum_{j=2}^{\infty}(B|v|)^{j}=|v|-\frac{B^{2}|v|^{2}}{1-B|v|}=|v|\left(1-\frac{B^{2}|v|}{1-B|v|}\right) .
$$

Letting $|v| \leq 1 /\left(2 B^{2}\right)<1 / 4 B$, we see that $|T v| \geq|v| / 3$. The proposition now follows (with $g=1 / 3 B)$ from this and the openness of the maps $T_{p}$.

Of course, since $d T_{p}=\mathrm{id}$, the Inverse Function Theorem says that $T_{p}$ is invertible on a neighborhood of $0_{p}$. The next proposition shows that if $T$ is slowly varying then so is the size of this neighborhood.

Proposition 4.2. Let $T / \mathrm{id}$ be as above. Then there exists a slowly varying function $h: X \rightarrow(0,1)$ such that $T_{p}$ is well-defined and injective on $E_{p}(h(p))$ for each $p \in X$. 
Proof. Let $h$ be a function to be specified shortly. It is easily shown (say, using linear coordinates on $E_{p}$ ) that there is a constant $C$, depending only on the rank of $E$, such that for all $j \geq 2, p \in X$, and $x, y \in E_{p}(h(p))$,

$$
\left|T_{j}(x)-T_{j}(y)\right| \leq C(j+1)^{k} B(p)^{j} h(p)^{j-1}|x-y|,
$$

where $k=\operatorname{rank}(E)$. Now

$$
|T(x)-T(y)|=\left|x-y+\sum_{j=2}^{\infty}\left(T_{j}(x)-T_{j}(y)\right)\right| \geq|x-y|\left(1-\sum_{j=2}^{\infty} C(j+1)^{k} B(B h)^{j-1}\right) .
$$

Thus $h=1 /\left(2 B^{3}\right)$ does the trick provided $B$ is bounded from below by a sufficiently large constant. This completes the proof.

Contracting linear maps. We say that a linear bundle map $A$ / $\phi$ of a bundle $E \rightarrow X$ is contracting if there exists $\lambda<0$ such that $\lim \sup _{N \rightarrow \infty} \frac{1}{N} \log \left|A^{N} v\right| \leq \lambda$ for every $v \in E$. If $A$ is slowly varying, then this implies that there is a slow variation $R_{\epsilon}: X \rightarrow(1, \infty)$ such that

$$
\left\|A_{p}^{N}\right\| \leq R_{\epsilon}(p) e^{(\lambda+\epsilon) N} \quad p \in X, N \geq 1
$$

There is a standard way of making this contraction more uniform by changing the metric. To this end, fix $\epsilon>0$ and set

$$
\langle v, w\rangle^{*}:=\sum_{N=0}^{\infty} e^{-2(\lambda-\epsilon) N}\left\langle A^{N} v, A^{N} w\right\rangle, \quad v, w \in E
$$

and denote the associated norm by $|\cdot|^{*}$ and operator norm by $\|\cdot\|^{*}$. Using (4.3) it is straightforward to verify that the series (4.4) converges, and that the metric thus obtained is a Borel metric on $E$ with the following properties: $\|A\|^{*} \leq e^{\lambda+\epsilon}$ and there exists an $\epsilon$-slowly varying function $C: X \rightarrow(1, \infty)$ such that

$$
|v| \leq|v|^{*} \leq C(p)|v|, \quad v \in E_{p}
$$

Splittings and flags. Suppose that the vector bundle $E \rightarrow X$ splits, i.e. there exist subbundles $E_{1}, \ldots, E_{l}$ of $E$ such that

$$
E=\bigoplus_{j=1}^{l} E_{j}
$$

We say that this splitting is slowly varying if the projection maps $E \rightarrow E_{j}$ are all slowly varying. It is possible to show that the splitting $E=\oplus E_{j}$ is slowly varying if and only if the angle functions $\measuredangle\left(E_{i}, E_{j}\right)$ are slowly varying functions.

To a splitting $\oplus E_{i}$ there is an associated flag $V_{\bullet}$, i.e. a sequence $V_{0}=\{0\} \subsetneq V_{1} \subsetneq \cdots \subsetneq V_{l}=E$ of vector subbundles defined by

$$
V_{j}=\bigoplus_{i=1}^{j} E_{i}
$$

Since this flag comes from a splitting, it is equipped with projections $\operatorname{pr}_{j}: E \rightarrow V_{j}$. We remark that the flag $V_{\bullet}$ depends on the ordering of the vector spaces $E_{i}$. For our applications, the $E_{i}$ are Lyapunov spaces and the Lyapunov exponents provide a natural ordering. 
Pseudo-linear maps. Let $E \rightarrow X$ be a vector bundle with a splitting $E=\oplus E_{j}$. A homogeneous selfmap $\Phi$ of degree $m$ of $E$ can then be further decomposed as

$$
\Phi=\sum_{j=1}^{l} \sum_{|\alpha|=m} \Phi_{j, \alpha}
$$

where

$$
\Phi_{j, \alpha}: E \rightarrow E_{j} \quad \text { and } \quad \Phi_{j, \alpha}(c \cdot v)=c^{\alpha} \Phi_{j, \alpha}(v) .
$$

Here $c=\left(c_{1}, \ldots, c_{l}\right) \in \mathbf{C}^{l}, \alpha=\left(\alpha_{1}, \ldots, \alpha_{l}\right) \in \mathbf{N}^{l}, c^{\alpha}=c_{1}^{\alpha_{1}} \ldots c_{l}^{\alpha_{l}}$ and

$$
c \cdot\left(v_{1} \oplus \cdots \oplus v_{l}\right)=c_{1} v_{1} \oplus \cdots \oplus c_{l} v_{l} .
$$

It follows easily from the definition that if $\Phi$ is a slowly varying homogeneous map of $E$, then all of the summands $\Phi_{j, \alpha}$ in the decomposition (14.5) are slowly varying.

A selfmap $\Phi$ of $E$ is said to be a flag map (with respect to the flag $V_{\bullet}$ associated to $\oplus E_{j}$ ) if $\operatorname{pr}_{j} \Phi$ is a map of $V_{j}$ for all $j$, i.e.

$$
\Phi\left(v_{1} \oplus \cdots \oplus v_{l}\right)=\Phi_{1}\left(v_{1}\right) \oplus \Phi_{2}\left(v_{1} \oplus v_{2}\right) \oplus \cdots \oplus \Phi_{l}\left(v_{1} \oplus \cdots \oplus v_{l}\right) .
$$

We shall say that a flag map $\Phi$ is pseudo-linear if it is of the form

$$
\Phi=A+H,
$$

where $A$ is a linear map which preserves the splitting, and $H$ is a polynomial flag map with no constant or linear part, such that $\operatorname{pr}_{j} H=\operatorname{pr}_{j} H \mid V_{j-1}$ for $2 \leq j \leq l$. In other words, $\Phi$ can be written

$$
\Phi\left(v_{1} \oplus \cdots \oplus v_{l}\right)=A v_{1} \oplus\left(A v_{2}+H_{2}\left(v_{1}\right)\right) \oplus \cdots \oplus\left(A v_{l}+H_{l}\left(v_{1} \oplus \cdots \oplus v_{l-1}\right)\right) .
$$

Notice that if $A$ is invertible, then so is $\Phi$, and its inverse is a pseudo-linear flag map whose degree is bounded in terms of the degree of $\Phi$.

Contracting pseudo-linear maps. A bundle map $\Phi / \phi$ of a bundle $E \rightarrow X$ is said to be contracting if its linear part $d \Phi \mid O_{E}$ is contracting. If $\Phi$ is slowly varying, then this implies that for $v \in E$ with $|v|$ small one has $\left|\Phi^{N} v\right| \rightarrow 0$ as $N \rightarrow \infty$. On the other hand, if $\Phi$ is linear (and contracting), then this convergence holds locally uniformly for $v \in E$. The next result, which will be a crucial ingredient in the proof of Theorem 2 shows that the same property carries over to some pseudo-linear maps $\Phi$.

Proposition 4.3. Let $E \rightarrow X$ be a measurable bundle with a slowly varying splitting $\oplus E_{i}$ and associated flag $V_{\bullet}$. Let $\Phi / f$ be a slowly varying, pseudo-linear, contracting bundle map with respect to $V_{\bullet}$. Then $\Phi^{N} \rightarrow 0$ locally uniformly in the topology on $E$. More precisely, there exists $\lambda<0$ with the following property: if $p \in X$ and $J \subset \subset E_{p}$ then there exists $C=C(J, p)>0$ such that

$$
\left|\Phi^{N} v\right| \leq C e^{\lambda N} \quad \text { for all } v \in J \text { and } N \geq 0 \text {. }
$$

Proof. The statement of the proposition does not change if we replace the metric on $E$ by the metric given by (4.4), so let us work with that metric. By compactness of $J$ it suffices to show the estimate (4.7) for large $N$.

Let $\Phi=A+H$ as in the definition of pseudo-linear, and let $\lambda<0$ be the associated exponent of contraction. Thus we have $\|A\| \leq e^{\lambda}$ on $E_{f^{N} p}$ for all $N \geq 0$. Pick $\epsilon>0$ so small that $\lambda+3 \epsilon<0$.

For $N \geq 1$ let $\Phi^{N}=\Phi_{1}^{N} \oplus \cdots \oplus \Phi_{l}^{N}$ be the decomposition of $\Phi^{N}: E \rightarrow E$ relative to the splitting $E=E_{1} \oplus \cdots \oplus E_{l}$. Then $\Phi_{1}^{N}=A^{N}$ so $\left\|\Phi_{1}^{N}\right\| \leq e^{\lambda N}$. For $2 \leq j \leq l$ we have

$$
\Phi_{j}^{N+1}=A \Phi_{j}^{N}+H_{j}\left(\Phi_{1}^{N} \oplus \cdots \oplus \Phi_{j-1}^{N}\right),
$$


where the $H_{j}$ 's are polynomials with no constant or linear terms. The slowly varying nature of the $H_{j}$ 's implies the existence of constants $r_{0}>0$ and $C_{0}>0$ such that

$$
\left|H_{j}(w)\right| \leq C_{0} e^{\epsilon N}|w|^{2} \quad \text { whenever } w \in E_{f^{N} p} \text { and }|w| \leq r_{0} e^{-\epsilon N} .
$$

We now inductively show the following estimate, which clearly implies the statement of the proposition (with $\lambda$ replaced by $\lambda+\epsilon$ ): there exists $N_{0}>0$ such that if $v \in J, 1 \leq i \leq l$ and $N \geq N_{0}$ then

$$
\left|\Phi_{i}^{N} v\right| \leq e^{(\lambda+\epsilon) N} \ll r_{0} e^{-\epsilon N} .
$$

This estimate clearly holds for $i=1$. Suppose it holds for $1 \leq i<j \leq l$ and let us show that it then hold for $i=j$ after possibly increasing $N_{0}$. Indeed, if $N \geq N_{0}$ then (4.8), (4.9) and (4.10) imply that

$$
\left|\Phi_{j}^{N+1} v\right| \leq e^{\lambda} e^{(\lambda+\epsilon) N}+C_{0} e^{\epsilon N} e^{2(\lambda+\epsilon) N} \leq e^{(\lambda+\epsilon)(N+1)}
$$

if $N_{0}$ is large enough. Thus (4.10) holds, which completes the proof.

Regular bundle maps. Consider a bundle $E \rightarrow X$ with a splitting $\oplus E_{j}$. A linear bundle map $A: E \rightarrow E$ is said to be regular if it preserves the splitting and if there exist $\lambda_{l}<\cdots<\lambda_{1}$ such that

$$
\lim _{N \rightarrow \infty} \frac{1}{N} \log \left|A^{N} v\right|=\lambda_{j} \quad \text { for } v \in E_{j}
$$

Now suppose, in addition, that the map $A$ and the splitting $\oplus E_{i}$ are slowly varying. Then one may show, using standard techniques as in [M], that there exists a slow variation $R_{\epsilon}: X \rightarrow(1, \infty)$ such that for all $p \in X$, all $\epsilon>0$, and all $N \in \mathbf{Z}$ :

$$
R_{\epsilon}(p)^{-1} e^{-\epsilon N} \leq \frac{\left|A_{p}^{N} v\right|}{e^{\lambda_{j} N}|v|} \leq R_{\epsilon}(p) e^{\epsilon N} \quad \text { whenever } \quad v \in E_{j, p}
$$

A general bundle map $\Phi / \phi$ is said to be regular if it is slowly varying and if the linear bundle map $A:=d F \mid 0_{E}$ is regular. The vector $\underline{\lambda}=\left(\lambda_{r}, \ldots, \lambda_{1}\right)$ is said to be the Lyapunov data associated to $\Phi$. It is easy to see that a regular bundle map is contracting if and only if its Lyapunov data satisfies $\lambda_{1}<0$.

The part of Oseledec's Theorem that we will use can be stated as follows. There exist invariant Borel sets $\mathcal{K}(\underline{\lambda}, \underline{m})$ in $M$, the union of which has total measure. Over each $\mathcal{K}(\underline{\lambda}, \underline{m})$ there is a measurable bundle $E^{s} \subset T M$ with a slowly varying splitting $\oplus E^{\lambda_{i}}$ such that $A:=d f \mid E^{s}$ is a regular contracting bundle map fibered over $f$.

The definition of a linear bundle map being regular is asymptotic in nature. The concept of an adapted metric makes this information easier to work with. For $\epsilon>0$ we define

$$
\langle v, w\rangle_{\epsilon}^{*}:=\sum_{N \in \mathbf{Z}} \frac{\left\langle A^{N} v, A^{N} w\right\rangle}{e^{2 \lambda_{j} N+2 \epsilon|N|}}, \quad v, w \in E_{j}
$$

and $\langle v, w\rangle_{\epsilon}=0$ for $v \in E_{i}, w \in E_{j}, i \neq j$. We denote the associated norm by $|\cdot|_{\epsilon}^{*}$ and operator norm by $\|\cdot\|_{\epsilon}^{*}$. Often we will drop the subscript and write $|\cdot|^{*}$ instead of $|\cdot|_{\epsilon}^{*}$.

Using (4.12 and the slow variation of the splitting $\oplus E_{j}$ it is not too hard to verify that the series (4.13) converges, and that the metric thus obtained is a Borel metric on $E$ with the following properties:

$$
e^{\lambda_{j}-\epsilon}|v|_{\epsilon}^{*} \leq|A v|_{\epsilon}^{*} \leq e^{\lambda_{j}+\epsilon}|v|_{\epsilon}^{*} \quad \text { for } v \in E_{j},
$$

and there exists a slow variation $C_{\epsilon}: X \rightarrow(1, \infty)$ such that

$$
|v| \leq|v|_{\epsilon}^{*} \leq C_{\epsilon}(p)|v|, \quad v \in E_{p} .
$$


Recall that a bundle map $\Phi / \phi$ is slowly varying if and only if the function $p \mapsto \sup _{m}\left\|\Phi_{m, p}\right\|^{1 / m}$ is slowly varying, where $\sum \Phi_{m}$ is the homogeneous expansion of $\Phi$. Using (4.14) it is straightforward to verify that $\Phi$ is slowly varying if and only if there exists a slow variation $R_{\epsilon}$ such that

$$
\left\|\Phi_{m . p}\right\|_{\epsilon}^{*} \leq R_{2 \epsilon}(p)^{m} .
$$

In this sense, working with the given metric $|\cdot|$ or the adapted metric $|\cdot|_{\epsilon}^{*}$ has no effect on the definition of slow variation.

\section{LOCAL ANALYTIC CONJUGATION}

The goal of this section is to establish the main step in the proof of Theorem 2, namely a local conjugation of regular bundle maps to a pseudo-linear model.

Theorem 5.1. Let $E \rightarrow X$ be a measurable bundle over an $f$-invariant Borel set $X \subset M$ with a slowly varying splitting $E=\oplus E_{j}$. Let $F / f$ be a slowly varying, contracting, and regular bundle map of $E$ (with respect to this splitting). Then there exists a polynomial bundle map $P / f$ with $d P\left|0_{E}=d F\right| 0_{E}$ and a bundle map $T /$ id with $d T \mid 0_{E}=\mathrm{id}$, so that

$$
T F T^{-1}=P
$$

holds on a slowly varying neighborhood of the zero section $O_{E}$ in $E$. Moreover, $P$ and $T$ are slowly varying, and $P$ is a pseudo-linear, contracting, polynomial automorphism of the flag associated to the splitting of $E$.

One should view $T$ as a conjugacy (i.e. change of coordinates) that conjugates $F$ to the simpler mapping $P$. The proof of Theorem 5.1 occupies the remainder of this section.

Proof of Theorem 5.1. Write $A=d F \mid O_{E}$ and recall our notation $O(m)$. We construct a pair of sequences $\left\{P_{m} / f\right\}_{m \geq 1}$ and $\left\{T_{m} / \text { id }\right\}_{m \geq 1}$ of slowly varying polynomial bundle maps such that:

(a) $T_{m}=$ id $+O(2)$ and $P_{m}=A+O(2)$;

(b) $P_{m}^{-1} T_{m} F-T_{m}=O(m+1)$;

(c) there exists $m_{0} \geq 1$ such that $P_{m}=P_{m_{0}}$ for all $m \geq m_{0}$;

(d) $P:=P_{m_{0}}$ is a pseudo-linear map of the flag associated to the splitting of $E$;

(e) $T_{m}$ converges to an analytic map $T$.

The construction is inductive, and proceeds as follows. Set $P_{1}=A$ and $T_{1}=$ id. Suppose that we have constructed $T_{m}$ and $P_{m}$. Let

$$
T_{m+1}=T_{m}+H_{m+1} \quad \text { and } \quad P_{m+1}=P_{m}\left(\mathrm{id}+Q_{m+1}\right),
$$

with $H_{m+1}$ and $Q_{m+1}$ to be determined. Then a simple calculation shows that

$$
P_{m+1}^{-1} T_{m+1} F-T_{m+1}=P_{m}^{-1} T_{m} F-T_{m}-\left(Q_{m+1}+H_{m+1}-A^{-1} H_{m+1} A\right)+O(m+2) .
$$

Thus, writing $\Phi_{m+1}:=P_{m}^{-1} T_{m} F-T_{m} \bmod O(m+2)$, we see that (b) holds for $m+1$ if we can find homogeneous solutions $H_{m+1}$ and $Q_{m+1}$ for the equation

$$
\Phi_{m+1}=Q_{m+1}+H_{m+1}-A^{-1} H_{m+1} A .
$$

The next lemma shows that solutions of (5.1) exist so that (c) and (d) hold as well.

Lemma 5.2. If $\Phi /$ id is a homogeneous, slowly varying, polynomial bundle mapping of degree $m \geq 2$, then there exist homogeneous, slowly varying polynomial bundle mappings $Q / \mathrm{id}$ and $H / \mathrm{id}$, also of degree $m$, such that

$$
\Phi=Q+H-A^{-1} H A .
$$


Moreover $Q$ can be chosen as follows. If $m$ is sufficiently large, then one can take $Q=0$. Otherwise, $Q$ is a pseudo-linear map of the flag associated to $\oplus E_{j}$ with no linear part, i.e. $Q$ has the form

$$
Q\left(v_{1} \oplus \cdots \oplus v_{l}\right)=0 \oplus Q_{2}\left(v_{1}\right) \oplus \cdots \oplus Q_{l}\left(v_{1} \oplus \cdots \oplus v_{l-1}\right) .
$$

Before proving Lemma 5.2, we need to develop a few ideas. First, the homogeneous polynomial bundle mapping $\Phi$ can be decomposed with respect to the splitting $\oplus E_{i}$ into $(j, \alpha)$-homogeneous parts $\Phi_{j, \alpha}$ as in (4.5). By linearity we only need to solve (5.2) for each summand $\Phi_{j, \alpha}$. Recall that if $\Phi$ is slowly varying, then so are these summands.

In order to solve (5.2) we will make crucial use of the fact that $A$ is regular with respect to the splitting $\oplus E_{i}$. Let $\underline{\lambda}=\left(\lambda_{1}, \ldots, \lambda_{l}\right)$ be the Lyapunov data associated to $A$ and notice that

$$
\lambda_{l}<\cdots<\lambda_{1}<0
$$

since $A$ is contracting. If $\Phi$ is $(j, \alpha)$-homogeneous, then it follows that

$$
\left\|A^{-N} \circ \Phi \circ A^{N}\right\| \sim e^{\left(\underline{\lambda} \cdot \alpha-\lambda_{j}\right) N}\|\Phi\| \text { for }|N| \gg 1 .
$$

This estimate, which is the key to the analysis in this section, will be made more precise below. Here we only note that the quantity $\lambda_{j}-\underline{\lambda} \cdot \alpha$ plays an obvious role in (5.3) and leads to the following definition. We say that the pair $(j, \alpha)$ is resonant if

$$
\lambda_{j}=\underline{\lambda} \cdot \alpha .
$$

It is called non-resonant otherwise, and more specifically, super-resonant or sub-resonant if we have $\lambda_{j}-\underline{\lambda} \cdot \alpha<0$ or $\lambda_{j}-\underline{\lambda} \cdot \alpha>0$, respectively.

Lemma 5.3. There exists $m_{0} \in \mathbf{N}$ such that all pairs $(j, \alpha)$ with $|\alpha|>m_{0}$ are sub-resonant. Furthermore, if $(j, \alpha)$ is resonant then $\alpha_{j}=\alpha_{j+1}=\cdots=\alpha_{l}=0$.

Proof. For the first statement pick $m_{0} \geq \lambda_{l} / \lambda_{1}$. The second statement is easily verified.

For the rest of this section we work with a fixed but small $\epsilon>0$. Specifically we require that

$$
\sup \left\{\underline{\lambda} \cdot \alpha-\lambda_{j}+(|\alpha|+2) \epsilon\right\}<0,
$$

where the supremum is taken over all sub-resonant pairs $(j, \alpha)$ (it is easy to check that this is possible), and

$$
\min \left\{\underline{\lambda} \cdot \alpha-\lambda_{j}-(|\alpha|+2) \epsilon\right\}>0
$$

where the minimum is taken over all sub-resonant pairs $(j, \alpha)$; the latter form a finite set by Lemma 5.3.

Lemma 5.4. With notation as in Lemma 5.9, if $(j, \alpha)$ is non-resonant and $\Phi$ is $(j, \alpha)$-homogeneous, then one can find a slowly varying $H /$ id so that

$$
\Phi=H-A^{-1} H A .
$$

Further, there exists a constant $C$, depending only on on the Lyapunov data $\underline{\lambda}$, such that

$$
\|H\|^{*} \leq C\|\Phi\|^{*}
$$

where $\|\cdot\|^{*}=\|\cdot\|_{\epsilon}^{*}$ is the operator norm associated to the adapted metric (4.13).

Proof. The operator $\Psi \mapsto \Psi-A^{-1} \Psi A$ has the following two formal inverses:

$$
\Psi \mapsto \mathcal{G}^{+}(\Psi):=\sum_{N=0}^{\infty} A^{-N} \Psi A^{N} \quad \text { and } \quad \Psi \mapsto \mathcal{G}^{-}(\Psi):=-\sum_{N=1}^{\infty} A^{N} \Psi A^{-N} .
$$

We proceed in two cases.

(i) Sub-resonant case: let $H=\mathcal{G}^{+}(\Phi)$.

(ii) Super-resonant case: let $H=\mathcal{G}^{-}(\Phi)$. 
We have to show that this makes sense. Let us consider the sub-resonant case (i). We have

$$
\begin{aligned}
\left\|\mathcal{G}^{+} \Phi\right\|^{*} & \leq \sum_{N=0}^{\infty}\left\|A^{-N} \Phi A^{N}\right\|^{*} \\
& \leq \sum_{N=0}^{\infty} e^{\left(\underline{\lambda} \cdot \alpha-\lambda_{j}+(|\alpha|+2) \epsilon\right) N}\|\Phi\|^{*} \\
& =: C\|\Phi\|^{*} .
\end{aligned}
$$

It follows from (5.4) that $C<\infty$ and that $C$ does not depend on $j, \alpha$ or $\epsilon$. Thus (5.6) holds, and this implies that $H$ is slowly varying. Notice that the construction of $H$ does not depend on the choice of $\epsilon$. The super-resonant case is treated similarly, using (5.5). This completes the proof.

Proof of Lemma 5.9. Simply decompose $\Phi$ as a sum of resonant, sub-resonant and super-resonant $(j, \alpha)$-homogeneous polynomial bundle mappings $\Phi_{j, \alpha}$. For the non-resonant terms we get $H_{j, \alpha}$ from Lemma 5.4 and set $Q_{j, \alpha}=0$. For the resonant terms we set $H_{j, \alpha}=0$ and $Q_{j, \alpha}=\Phi_{j, \alpha}$. Then set $H=\sum H_{j, \alpha}$ and $Q=\sum Q_{j, \alpha}$. From Lemma 5.4 we get that $H$ and $Q$ are slowly varying. The remaining statements follow from Lemma 5.3.

Returning to the proof of Theorem 5.1 we have constructed our sequences of maps $P_{m}$ and $T_{m}$, and have shown that they satisfy (a)-(d) above. It remains to show that $T=\lim T_{m}$ is analytic and slowly varying. For this it suffices to show that $\left\|H_{m}\right\|^{*} \leq B^{h(m)}$ for some $\epsilon$-slowly varying $B: X \rightarrow(1, \infty)$ and some concave function $h: \mathbf{N} \rightarrow \mathbf{R}_{+}$.

Recall that the degree of the inverse of a pseudo-linear map is bounded in terms of the degree of the map itself. In particular, the degree $\nu$ of $P^{-1}$ is finite, so we may write

$$
P^{-1}=S A^{-1}=\left(S_{1}+S_{2}+\cdots+S_{\nu}\right) A^{-1},
$$

where $S_{1}=$ id and $S_{j}$ is homogeneous of degree $j$, and all the maps are slowly varying. Finally, write $F=A G$, so that $G /$ id is analytic and slowly varying. We denote the homogeneous expansion of $G$ by

$$
G=\sum_{j=1}^{\infty} G_{j}
$$

observing that $G_{1}=$ id.

Keeping $\epsilon$ fixed we now pick an $\epsilon$-slowly varying $B: X \rightarrow(2, \infty)$ with the following properties:

$$
\begin{aligned}
\left\|S_{i}\right\|^{*} & \leq B^{\alpha} \text { for } 2 \leq i \leq \nu \\
\left\|H_{m}\right\|^{*} & \leq B^{\alpha} \text { for } 2 \leq m \leq m_{1} \\
\left\|G_{k}\right\|^{*} & \leq B^{k \alpha} \text { for } k \geq 2 \\
\left\|A^{-1}\right\|^{*} & \leq B^{\alpha} \text { and }\|A\|^{*} \leq 1 .
\end{aligned}
$$

Here $\alpha \in(0,1)$ and $m_{1}>m_{0}$ will be chosen later. As before we write $\|\cdot\|^{*}=\|\cdot\|_{\epsilon}^{*}$. That (5.8)-(5.11) are possible follows from the fact that each $S_{i}$ and each $H_{m}$ are slowly varying, as are $G$ and $A^{-1}$, while $A$ is contracting. We claim that if $\alpha$ is small enough and $m_{1}$ is large enough, then

$$
\left\|H_{m}\right\|^{*} \leq B^{m-\sqrt{m}} \text { for all } m \geq 2 .
$$

This is clearly true for $m \leq m_{1}$ by our choice of $B$. Suppose that (5.12) holds for some $m$. To establish it for $m+1$ it suffices, in view of Lemma 5.4, to prove that

$$
\left\|\Phi_{m+1}\right\|^{*} \leq C^{-1} B^{m+1-\sqrt{m+1}},
$$


where $\Phi_{m+1}$ is given by (5.1). To this end, let us take a look at the quantity $P^{-1} T_{m} F-T_{m}$, keeping in mind that we are only interested in the terms of degree $m+1$. Thus the terms coming from $-T_{m}$ automatically disappear, and we have

$$
\begin{aligned}
\Phi_{m+1} & =P^{-1} T_{m} F-T_{m} \bmod O(m+2) \\
& =\sum S_{i} A^{-1} H_{j} A G_{k},
\end{aligned}
$$

where the sum is over all $i, j$ and $k$ such that $i j k=m+1, k \geq 1,1 \leq j \leq(m+1) / 2$ and $1 \leq i \leq \nu$. (The constraint on the index $j$ comes from the fact that (i) $1 \leq j \leq m$, and (ii) we need only consider the terms of degree $m+1$.)

The sum (5.13) consists of considerably fewer than $\nu m^{2}$ terms. We now estimate $S_{i} A^{-1} H_{j} A G_{k}$ in all possible cases, using (5.8)-(5.12) and the induction hypothesis, and assuming that $\alpha$ is small while $m_{1}$ is large.

(i) $(k \geq 2, i \geq 2)$ : then $i j \leq(m+1) / 2$ and

$$
\left\|S_{i} A^{-1} H_{j} A G_{k}\right\|^{*} \leq B^{\alpha+i(\alpha+(j-\sqrt{j})+j k \alpha)} \leq B^{(m+1)\left(\frac{1}{2}+3 \alpha\right)} \leq \frac{1}{C \nu m^{2}} B^{m+1-\sqrt{m+1}} .
$$

(ii) $(k \geq 2, i=1)$ : then $j \leq(m+1) / 2$ and

$$
\left\|A^{-1} H_{j} A G_{k}\right\|^{*} \leq B^{\alpha+(j-\sqrt{j})+j k \alpha} \leq B^{(m+1)\left(\frac{1}{2}+2 \alpha\right)} \leq \frac{1}{C \nu m^{2}} B^{m+1-\sqrt{m+1}} .
$$

(iii) $(k=1)$ : then $i \geq 2$ and $i j=(m+1)$. Further, a straightforward calculation readily shows that $\sqrt{m+1}(\sqrt{i}-1)-\alpha(1+i) \geq \alpha \sqrt{m+1}$, so

$$
\begin{aligned}
\left\|S_{i} A^{-1} H_{j} A\right\|^{*} \leq B^{\alpha+i(\alpha+(j-\sqrt{j}))} & =B^{(m+1-\sqrt{m+1})+(1+i) \alpha-\sqrt{m+1}(\sqrt{i}-1)} \\
& \leq B^{(m+1-\sqrt{m+1})-\alpha \sqrt{m+1}} \\
& \leq \frac{1}{C \nu m^{2}} B^{m+1-\sqrt{m+1}} .
\end{aligned}
$$

Putting all this together, we have:

$$
\left\|\Phi_{m+1}\right\|^{*} \leq \sum_{i, j, k}\left\|S_{i} A^{-1} H_{j} A G_{k}\right\|^{*}<\nu m^{2} \frac{1}{C \nu m^{2}} B^{m+1-\sqrt{m+1}}=\frac{1}{C} B^{m+1-\sqrt{m+1}} .
$$

Thus the induction step is complete and (5.12) holds for all $m \geq 2$. Theorem 5.1 now follows from the definition of a slowly varying bundle map.

\section{Proof of Theorem 2}

We may replace $\mathcal{K}(f)$ by the Borel set $\mathcal{K}(\underline{\lambda}, \underline{m})$. Let us apply Theorem 5.1 to the bundle $E^{s} \rightarrow$ $\mathcal{K}(\underline{\lambda}, \underline{m})$ (with its Lyapunov splitting) and the bundle map $F=\chi^{-1} \circ f \circ \chi$ of $E^{s}$, where $\chi$ is the exponential map given by Theorem 3.4. By Theorem 3.2 and Theorem 3.4 the map $F$ is slowly varying, contracting and regular (with respect to the Lyapunov splitting). Thus we may apply Theorem 5.1 to $F$.

With $P$ and $T$ as in Theorem 5.1 let

$$
\Psi_{N}:=P^{-N} T \chi^{-1} f^{N}
$$

Then for each $p \in \mathcal{K}(\underline{\lambda}, \underline{m})$ and each compact subset $J \subset \subset W_{p}^{s}$ there exists an integer $N_{0}=N_{0}(J)$ such that $\Psi_{N}$ is well defined on $J$ whenever $N \geq N_{J}$. Indeed, since $f^{N}$ decays exponentially as $N \rightarrow \infty$, and $T \chi^{-1}$ is slowly varying, $f^{N}$ will carry $J$ into the (slowly varying) domain of $T \chi^{-1}$, provided $N$ is large enough. Then one has

$$
\Psi_{N+1}-\Psi_{N}=P^{-N}\left(P^{-1} T F-T\right) \chi^{-1} f^{N}=0 .
$$


It follows that, locally uniformly in the discrete topology on $\mathcal{W}^{s}, \Psi_{N}$ converges as $N \rightarrow \infty$ to a map $\Psi: \mathcal{W}^{s} \rightarrow E^{s}$. Evidently $\Psi$ is holomorphic on the fibers $W_{p}^{s}$ of $\mathcal{W}^{s}$, maps $W_{p}^{s}$ into $E_{p}^{s}$, and satisfies the functional equation

$$
P^{-1} \Psi f=\Psi .
$$

It remains only to show that $\Psi$ is bijective, which it obviously suffices to check on fibers. Thus we fix from here on a point $p \in \mathcal{K}(\underline{\lambda}, \underline{m})$.

First note that, by Proposition 4.2, there exists a slowly varying function $h$ such that $T \chi^{-1} \mid E^{s}(h)$ (and thus $P^{-N} T \chi^{-1} \mid E^{s}(h)$ ) is injective on fibers. If $x_{1}, x_{2} \in W_{p}^{s}$ are two points, then for large enough $N, f^{N} x_{j} \in E^{s}(h)$ for $j=1,2$. (Again, this is so because $f^{N}$ decays exponentially, and thus $f^{N} x_{j}$ eventually enters and remains inside the slowly varying tube $E^{s}(h)$.) But then, if $N$ is large enough,

$$
\Psi x_{1}=\Psi x_{2} \Rightarrow P^{-N} T \chi^{-1}\left(f^{N} x_{1}\right)=P^{-N} T \chi^{-1}\left(f^{N} x_{2}\right) \Rightarrow f^{N} x_{1}=f^{N} x_{2} \Rightarrow x_{1}=x_{2} .
$$

Thus $\Psi$ is injective.

Next, fix $v \in E_{p}^{s}$. We want to find $x \in W_{p}^{s}$ with $\Psi(x)=v$. By Proposition 4.1, there exists a slowly varying tube $E^{s}(g)$ in the range of $\Psi$. Further, by Proposition $4.3,\left|P^{N} v\right|$ decays exponentially, so $P^{N}(v)$ enters and remains inside $E^{s}(g)$ for large enough $N$. Let $y \in W_{f^{N} p}^{s}$ be such that $\Psi(y)=P^{N}(v)$ and set $x=f^{-N} y$. Then

$$
\Psi(x)=\Psi\left(f^{-N} y\right)=P^{-N} \Psi(y)=v,
$$

and thus $\Psi$ is surjective.

Finally, from the fact that $\left(d P_{p}\right)_{p}=A=d f_{p} \mid E^{s}$ and that $\left(d \chi_{p}\right)_{p}=$ id, we see from the definition of $\Psi=\Psi_{N}$ that $\left(d \Psi_{p}\right)_{p}=$ id. This completes the proof of Theorem 2 .

Final remarks. The proof of the existence of the normal form in the stationary case as presented in $[\mathrm{St}, \mathrm{RR}$ does not use the convergence of the formal solution $T$ of the local conjugation problem. Instead, one shows the existence of a sufficiently large integer $m$ such that $P^{-N} T_{m} F^{N}$ converges. This relies heavily on the fact that the iterates of a pseudo-linear map grow at most exponentially (see Lemma 1 in the Appendix of [RR]). This exponential growth seems quite hard to establish in the slowly varying setting - it would be interesting to know whether it is indeed possible. On the other hand, our method also provides a new proof in the analytic stationary case.

\section{REFERENCES}

[B] Bedford, E., Open problem session of the Biholomorphic Mappings meeting at the American Institute of Mathematics, Palo Alto, CA, July 2000.

[BLS] Bedford, E., Lyubich, M., Smillie, J., Polynomial diffeomorphisms of $\mathbf{C}^{2} I V$ : The measure of maximal entropy and laminar currents Invent. Math. 112 (1993), 77-125.

[BD] Briend, J.-Y., Duval, J., Exposants de Liapounoff et distribution des points périodiques d'un endomorphisme de $\mathbf{C P}^{k}$. Acta Math. 182 (1999), no. 2, 143-157.

[Br] Brown, M. The monotone union of open n-cells is an open n-cell Proc. Amer. Math. Soc. $121961812-814$.

[F] Fornæss, J.-E., An increasing sequence of Stein manifolds whose limit is not Stein. Math. Ann. 223 (1976), no. 3, 275-277.

[G] Guysinsky, M. The theory of non-stationary normal forms. Preprint.

[GK] Guysinsky, M., Katok, A., Normal forms and invariant geometric structures for dynamical systems with invariant contracting foliations. Math. Res. Lett. 5 (1998), no. 1-2, 149-163.

[M] Mañé, R., Ergodic theory and differentiable dynamics. Ergebnisse der Mathematik und ihrer Grenzgebiete (3), 8. Springer-Verlag, Berlin-New York, 1987

[O] Oseledec, V. A multiplicative ergodic theorem. Characteristic Ljapunov, exponents of dynamical systems. (Russian) Trudy Moskov. Mat. Obšč. 19 (1968) 179-210. English Transl. Trans. Moscow Math. Soc. 19 (1968), 197-231.

[RR] Rosay, J.P., Rudin, W. Holomorphic Maps from $\mathbf{C}^{n}$ to $\mathbf{C}^{n}$. Trans. AMS 310, 47-86 (1988).

[P] Pesin, Ya. Families of invariant manifolds that correspond to nonzero characteristic exponents. (Russian) Izv. Akad. Nauk SSSR Ser. Mat. 40 (1976), no. 6, 1332-1379, 1440. 
[PS] Pugh, C., Shub, M., Ergodic attractors. Trans. Amer. Math. Soc. 312 (1989), no. 1, 1-54.

[S] Shub, M., Global Stability of Dynamical Systems. Springer Verlag, 1987.

[Si] Siegel, C. L., Über die Normalform analytischer Differentialgleichungen in der Nähe einer Gleichgewichtslö sung. (German) Nachr. Akad. Wiss. Göttingen. Math.-Phys. Kl. Math.-Phys.-Chem. Abt. 1952, (1952). 21-30.

[St] Sternberg, S., Local contractions and a theorem of Poincaré. Amer. J. Math. 791957 809-824.

[W] Wu, H., Complex stable manifolds of holomorphic diffeomorphisms. Indiana Univ. Math. J. 42 (1993) 1349-1358.

Department of Mathematics; University of Michigan; Ann Arbor, Mi 48109-1109 